\section{Cureus}

\title{
Streptococcus Gordonii Empyema: A Case Report and Review of Empyema
}

Amanda M. Dave ${ }^{1}$, Felicia Ratnaraj ${ }^{2}$, Manasa Velagapudi ${ }^{3}$, Mridula Krishnan ${ }^{2}$, Nagarjuna R. Gujjula $^{2}$, Pamela A. Foral ${ }^{4}$, Laurel Preheim ${ }^{2}$

1. School of Medicine, Creighton University Medical Center 2. Internal Medicine, Creighton University Medical Center 3. Infectious Diseases, Creighton University Medical Center, Omaha, USA 4. Pharmacy Practice, Creighton University School of Pharmacy and Health Professions, Omaha, USA

$\square$ Corresponding author: Amanda M. Dave, amandakrantz@creighton.edu Disclosures can be found in Additional Information at the end of the article

\section{Abstract}

Streptococcus gordonii (S. gordonii) is a pioneer oral bacteria that is recognized as an agent of bacterial endocarditis. However, an extensive review of the literature revealed no reported case of $S$. gordonii causing empyema. We present a case of a 65 -year-old male who presented with respiratory distress. Physical examination revealed several dental caries with decreased breath sounds in the bibasilar regions. A computed tomography (CT) scan of the chest and abdomen demonstrated left-sided pleural effusion and a $4.3 \mathrm{~cm}$ x $2.8 \mathrm{~cm}$ splenic abscess. He received intravenous (IV) antibiotics, and his blood cultures remained negative. Drainage of the splenic abscess grew $S$. gordonii. A CT-guided thoracentesis yielded $450 \mathrm{ml}$ of exudative fluid. Pleural fluid cultures grew $S$. gordonii. A CT scan of the head and neck ruled out an intra-oral abscess. He received six weeks of IV penicillin with a follow-up CT scan showing resolution of both the splenic abscess and the left parapneumonic effusion.

Categories: Internal Medicine, Gastroenterology, Infectious Disease

Keywords: streptococcus gordonii, empyema, splenic abscess

\section{Introduction}

Viridans streptococci, including Streptococcus gordonii (S. gordonii), are pioneer oral bacteria often associated with dental plaque formation. $S$. gordonii is a gram-positive, non-motile cocci. It is a facultative anaerobe. $S$. gordonii has been well established as an inhabitant of the mouth and as an agent of endocarditis.

In the pre-antibiotic era, $S$. pneumoniae, $S$. pyogenes, and $S$. aureus were the most common pathogens associated with empyema. However, anaerobes have also been identified in a significant number of empyemas either as sole organisms or in mixed cultures. S. gordonii has not previously been reported as a cause of empyema.

\section{(C) Copyright 2017}

Dave et al. This is an open access article distributed under the terms of the Creative Commons Attribution License CC-BY 3.0., which permits unrestricted use, distribution, and reproduction in any medium, provided the original author and source are credited.

\section{Case Presentation}

A 65-year-old Caucasian male with a history of tobacco abuse, diabetes mellitus, and depression was admitted to a community hospital with hypotension, fever, and respiratory distress. Initial laboratory evaluations performed at the community hospital were within normal reference ranges and did not reveal leukocytosis. Chest radiographs revealed left middle lobe pneumonia so he was started on intravenous (IV) levofloxacin, $500 \mathrm{mg}$ daily, with a transition to oral levofloxacin, $500 \mathrm{mg}$ daily, for one week. He was transferred to a tertiary care center when his fever did not resolve and he developed new symptoms of abdominal pain. Informed 


\section{Cureus}

consent was obtained upon transfer.

On presentation, he was in significant respiratory distress requiring intubation, mechanical ventilation, and vasopressors. Physical examination revealed several dental caries with missing teeth and a draining socket. Decreased breath sounds were noted in the bibasilar regions. A computed tomography (CT) of the chest and abdomen demonstrated left-sided pleural effusion and a splenic fluid collection measuring $4.3 \mathrm{~cm}$ x $2.8 \mathrm{~cm}$ was clinically suspicious for a splenic abscess (Figure 1).

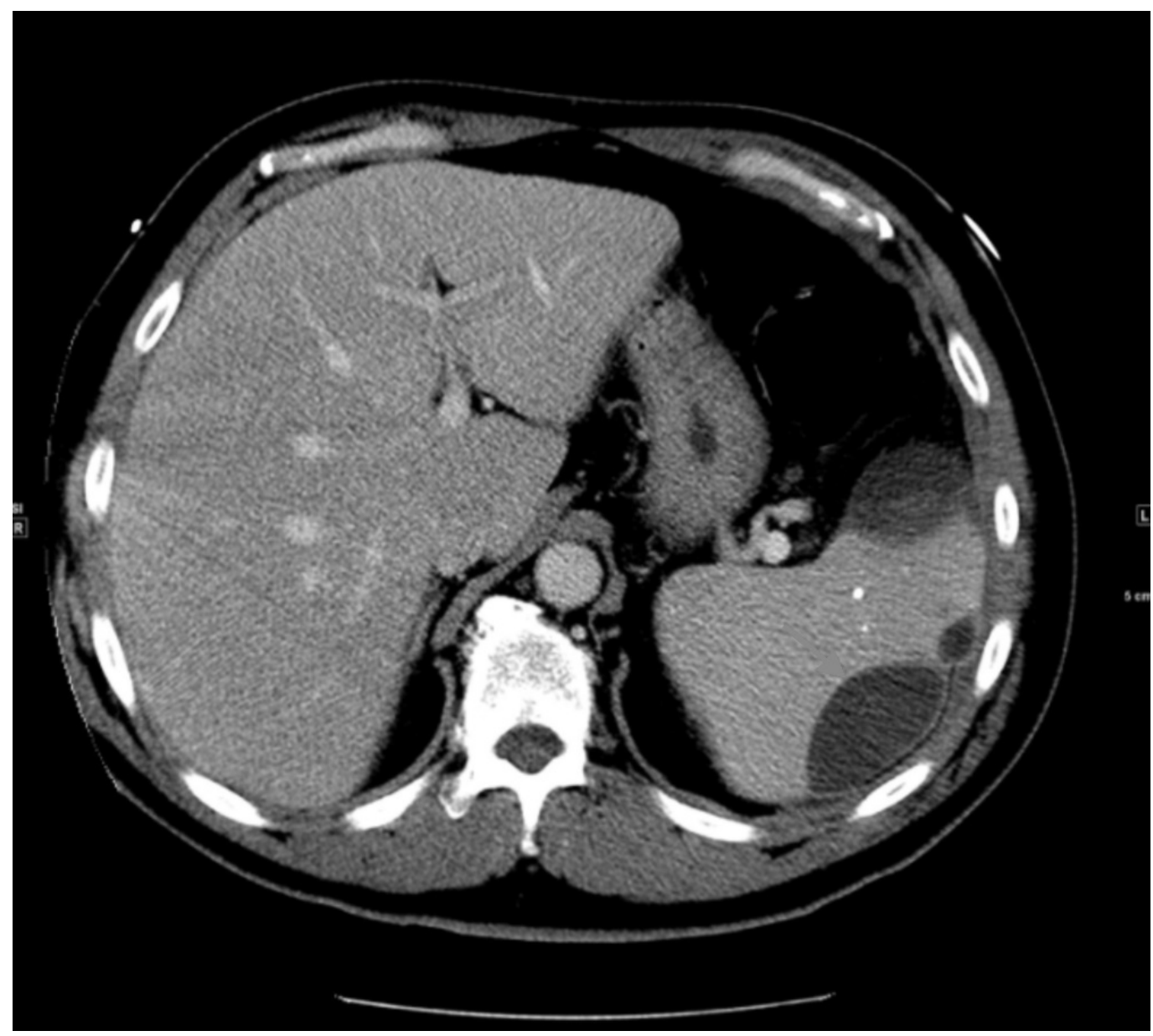

\section{FIGURE 1: Splenic Abscess}

A computed tomography (CT) of the chest and abdomen demonstrated a splenic fluid collection measuring $4.3 \mathrm{~cm} \times 2.8 \mathrm{~cm}$, clinically suspicious for an abscess.

He received weight-based dosing of IV vancomycin and piperacillin-tazobactam. Blood cultures were negative, procalcitonin was $6.51 \mathrm{ng} / \mathrm{ml}$ and lactic acid was $0.9 \mathrm{mmol} / \mathrm{L}$. Drainage of the empyema was performed and cultures yielded a rare species, $S$. gordonii, susceptible to penicillin, vancomycin, ceftriaxone, clindamycin, erythromycin, and levofloxacin. The sensitivities were as follows: sensitivity to penicillin (minimum inhibitory concentration (MIC) 0.094), vancomycin (MIC 1), ceftriaxone (MIC 0.38), clindamycin (MIC 0.023), erythromycin (MIC 0.023), and levofloxacin (MIC 0.5). A CT-guided thoracentesis yielded $450 \mathrm{ml}$ of exudative fluid with 4,329 nucleated white blood cells/mm 3 (79\% neutrophils). The culture of the splenic abscess also grew $S$. gordonii. A CT scan of the head and neck, including maxillofacial views, was negative for odontogenic infection, neck abscess, or significant cervical lymphadenopathy. 
The patient received six weeks of IV penicillin, 20 million units daily. Follow-up CT scan after treatment showed resolution of both the splenic abscess and the left parapneumonic effusion. He improved clinically and tolerated the medication without any side effects. After discharge, the patient was maintained on $875 \mathrm{mg}$ of amoxicillin twice daily for 10 months per recommendation from the infectious disease team.

\section{Discussion}

S. gordonii is a gram-positive, non-motile, cocci, facultative anaerobe member of the viridans group [1]. S. gordonii is commonly found in the mouth and is capable of spreading to extraoral sites, causing systemic infection [1]. S. gordonii, along with $S$. sanguinis and S. oralis, is commonly found in blood cultures of patients with infective endocarditis [2]. It is capable of colonizing platelet-fibrin thrombi on abnormal heart valves or the endocardium. It has also been reported in a case series of septic arthritis [3]. However, an extensive review of the literature revealed this to be the first case of $S$. gordonii empyema to be reported.

Empyema should be suspected in any new onset of pleural effusion in a patient who has had recent pneumonia, malignancy, or tuberculosis. An empyema can also follow a penetrating chest trauma or extension from a sub-diaphragmatic or paravertebral abscess [4]. Early diagnosis and complete drainage are critical as patients with empyema have higher rates of hospital admission, prolonged hospital stays, and increased mortality [5].

Radiographic imaging plays a key role in the evaluation and management of empyema [6]. Optimal evaluation of empyema requires a chest CT scan with contrast [7]. The criteria to consider thoracentesis is a thickened parietal pleura on a contrast-enhanced CT scan, a finding suggestive of empyema [6]. Based on the clinical presentation, it is difficult to ascertain whether the splenic abscess was secondary to the empyema or vice versa. The novelty of this case lies in it being the first known presentation of $S$. gordonii in an empyema.

Fluid obtained by thoracentesis should undergo microbiologic analysis with appropriate stains and cultures. Samples of the fluid should also be sent for a cell count with differential and chemistries; $\mathrm{pH}$ should be determined with a blood gas analyzer [8]. Pleural fluid with a $\mathrm{pH}<$ 7.20 suggests drainage per the consensus guidelines from the American College of Chest Physicians [8]. Early surgical consultation is required because the majority of these patients will require thoracoscopic intervention or surgical debridement [9].

Tube thoracostomy, video-assisted thoracoscopic surgery (VATS), open decortication, and open thoracostomy are the four drainage options for empyema. Tube thoracostomy is the most commonly preferred and the least invasive method of drainage of thoracic empyema $[4,10]$.

Complete and appropriate management of thoracic empyema includes systemic antibiotic therapy, adequate pleural fluid drainage, and obliteration of the empyema [10]. Empiric therapy should include agents effective against the oral and upper airway aerobic and anaerobic flora. The optimal duration of therapy often ranges from four to six weeks of IV antibiotics; some patients may require a longer antibiotic course. Adequate pleural fluid drainage is demonstrated by a minimal chest tube output and repeat imaging by a CT scan indicating the lack of any large residual loculations.

\section{Conclusions}

S. gordonii is a gram-positive, non-motile, facultative anaerobe member of the viridans group streptococci and is most commonly found in the mouth. This case represents the first known presentation of $S$. gordonii in an empyema. Our patient presented with unique complications of an S. gordonii infection, namely, an empyema and a splenic abscess. Empyema fluid is typically 
purulent, has a $\mathrm{pH}<7.20$, and contains bacteria identified on gram stain and/or cultures. Appropriate therapy of an empyema includes adequate drainage and antimicrobial therapy directed against the identified pathogen(s).

\section{Additional Information \\ Disclosures}

Human subjects: Consent was obtained by all participants in this study. Conflicts of interest: In compliance with the ICMJE uniform disclosure form, all authors declare the following:

Payment/services info: All authors have declared that no financial support was received from any organization for the submitted work. Financial relationships: All authors have declared that they have no financial relationships at present or within the previous three years with any organizations that might have an interest in the submitted work. Other relationships: All authors have declared that there are no other relationships or activities that could appear to have influenced the submitted work.

\section{References}

1. Kilian M, Mikkelsen L, Henrichsen J: Taxonomic study of viridans streptococci: description of Streptococcus gordonii sp. nov. and emended descriptions of Streptococcus sanguis (White and Niven 1946), Streptococcus oralis (Bridge and Sneath 1982), and Streptococcus mitis (Andrewes and Horder 1906). Int J Syst Evol Microbiol. 1989, 39:471-84. 10.1099/0020771339-4-471

2. Liao $\mathrm{CY}, \mathrm{Su} \mathrm{KJ}$, Lin $\mathrm{CH}$, et al.: Purpura as the initial presentation of viridians streptococcal shock syndrome secondary to streptococcus gordonii bacteremia. Can J Infect Dis Med Microbiol. 2016, 2016:9463895. 10.1155/2016/9463895

3. Yombi JC, Belkhir L, Jonckheere S, et al.: Streptococcus gordonii septic arthritis: two cases and review of literature. BMC Infect Dis. 2012, 12:215. 10.1186/1471-2334-12-215

4. Hirshberg B, Sklair-Levi M, Nir-Paz R, et al.: Factors predicting mortality of patients with lung abscess. Chest. 1999, 115:746-50. 10.1378/chest.115.3.746

5. Dean NC, Griffith PP, Sorensen JS, et al.: Pleural effusions at first ED encounter predict worse clinical outcomes in patients with pneumonia. Chest. 2016, 149:1509-15.

10.1016/j.chest.2015.12.027

6. Heffner JE, Klein JS, Hampson C: Diagnostic utility and clinical application of imaging for pleural space infections. Chest. 2010, 137:467-79. doi:10.1378/chest.08-3002

7. Tsujimoto N, Saraya T, Light RW, et al: A simple method for differentiating complicated parapneumonic effusion/empyema from parapneumonic effusion using the split pleura sign and the amount of pleural effusion on thoracic CT. PLoS One. 2015, 10:e0130141. doi:10.1371/journal.pone.0130141

8. Heffner JE, Brown LK, Barbieri C, DeLeo JM: Pleural fluid chemical analysis in parapneumonic effusions. A meta-analysis. Am J Respir Crit Care Med. 1995, 151:1700-8. 10.1164/ajrccm.151.6.7767510

9. Scarci M, Abah U, Solli P, et al.: EACTS expert consensus statement for surgical management of pleural empyema. Eur J Cardiothorac Surg. 2015, 48:642-53. 10.1093/ejcts/ezv272

10. Wozniak CJ, Paull DE, Moezzi JE, et al.: Choice of first intervention is related to outcomes in the management of empyema. Ann Thorac Surg. 2009, 87:1525-30.

doi:10.1016/j.athoracsur.2009.01.028 